\title{
Optimum brightness of a new light-emitting diode lightwand device in a cadaveric model - a pilot study
}

\author{
Andrew D. Milne, MD • Matthew I. d'Entremont, MASc $\cdot$ Orlando R. Hung, MD
}

Received: 15 December 2015/Revised: 21 December 2015/Accepted: 23 December 2015/Published online: 20 January 2016

(c) Canadian Anesthesiologists' Society 2016

\section{To the Editor,}

Lightwand devices have played an important role in management of the difficult airway. ${ }^{1}$ The recent withdrawal of the Trachlight ${ }^{\mathrm{TM}}$ from the market has left many anesthesia providers without a suitable replacement for this well-validated device. ${ }^{1}$ Despite its overall utility, the prior Trachlight was not without its limitations. It was known to have appreciable degradation in light output after repeated cleanings, ${ }^{2}$ be associated with a risk for thermal burns from the incandescent bulb, and a fixed brightness of $2282 \operatorname{lux}^{2}$ that could not be adjusted to account for varying patients' neck sizes. ${ }^{3}$

We recently developed a new prototype lightwand device with red and white light-emitting diode (LED) light sources and adjustable brightness. In this pilot study, we assessed the optimum light intensity and LED bulb colour (white $v s$ red) required for transtracheal illumination with this new device.

Our local institutional research ethics board granted approval (July 2012) for this study. Fourteen anesthesia staff members familiar with lightwand intubation consented to participate in the study. One was excluded from the study because of a technical problem during testing. One female and one male, minimally preserved,

A. D. Milne, MD $(\varangle) \cdot$ O. R. Hung, MD

Department of Anesthesia, Pain Management and Perioperative Medicine, Dalhousie University, Halifax, NS, Canada

e-mail: admilne@dal.ca

A. D. Milne, MD

School of Biomedical Engineering, Dalhousie University, Halifax, NS, Canada

M. I. d'Entremont, MASc

iDLab - Innovation in Design Laboratory, Dalhousie University,

Halifax, NS, Canada clinical-grade cadavers (neck circumferences of $38 \mathrm{~cm}$ and $40 \mathrm{~cm}$, respectively) were used for this pilot study. The brightness of the prototype device was controlled via a dimmer dial on a custom-built power supply. Two lightwand stylets were equipped with either a variablebrightness red (0-4500 lux) or white (0-8000 lux) LED bulb. The LED light output was calibrated using a previously described lightmeter and testing system. ${ }^{2}$ Each lightwand was placed inside a 7-mm endotracheal tube (Mallinckrodt Hi-Lo cuffed tracheal tube, Covidien) so the LED bulb did not protrude beyond the tip of the tube. It was bent to form a "hockey stick" configuration. ${ }^{1}$ The cadavers were intubated by one of the investigators with the endotracheal tube/lightwand tip placed in the glottis opening (confirmed by direct laryngoscopy). Testing was done with the room lighting darkened to $<3$ lux. For each bulb colour, the participants adjusted the dial to the optimum transtracheal illumination to confirm tracheal placement. These data were recorded in two ways, both from the fully off position up to the optimum level and from the maximum brightness down to the optimum level. ${ }^{4}$ The order of the testing scenario was block randomized. After testing was complete, the participants were asked which bulb colour they preferred. The optimum brightness levels were compared using analysis of variance followed by post-hoc Holm-Sidak testing. Two-sided $P<0.05$ (adjusted for multiple comparisons) was considered significant.

The optimum light intensity selected by the 13 staff participants for the different testing scenarios is shown in the Figure. There were significant differences in the optimum light intensity for the female $v s$ male cadavers $(P<0.001)$ and the red $v s$ white LED bulb brightness $(P<$ $0.001)$. There was no significant difference, however, in the 


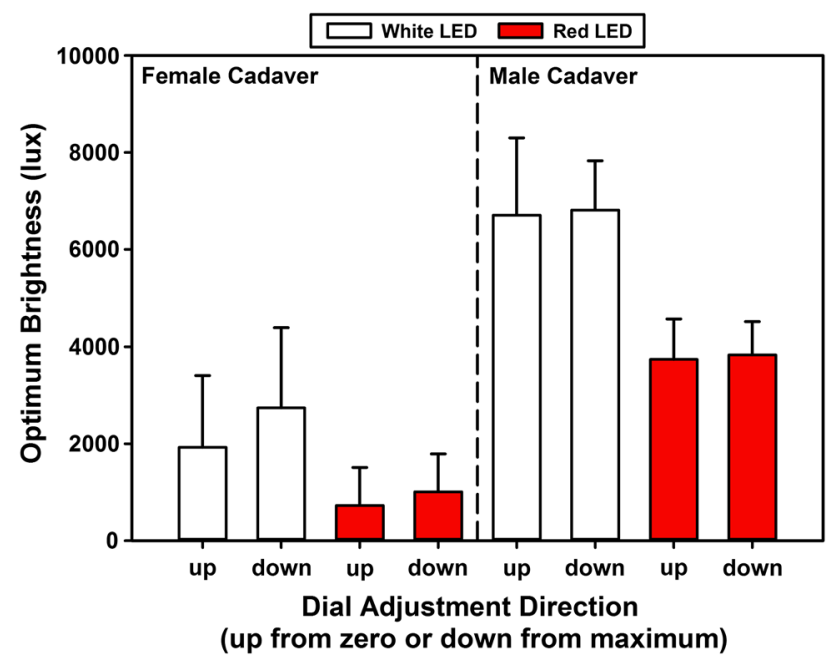

Figure Mean (SD) optimum light levels for transtracheal illumination. The $\mathrm{x}$-axis indicates the direction in which the light was adjusted by the staff anesthesiologists (i.e., gradually increasing from the "off" position or decreasing from the maximum brightness setting). Left: female cadaver. Right: male cadaver

optimum brightness selection by direction of dial adjustment $(P=0.16)$. The pattern of LED colour preference was variable, with $31 \%$ preferring the white LED light, $23 \%$ preferring the red LED, and $31 \%$ with no colour preference. The other $15 \%$ reported that their choice (either white or red) depended on the perceived neck thickness.

The optimum brightness required for transtracheal illumination was highly dependent on the anatomy of the cadaver tested, with a wide range of lux required for both LED bulb colours. The optimum brightness selected by the participants was up to threefold higher for the white LED and up to 1.7-fold higher for the red LED in comparison to the brightness of the prior Trachlight device. ${ }^{2}$

This study was limited in that we tested only two cadavers. Although cadavers are more realistic than plastic manikins as an experimental airway model, they may not fully represent the in vivo situation because the cadaver preservation process or lack of blood flow may influence light transmission properties in tissues.

The data from this study support use of the new lightwand device design with an adjustable light intensity and the option of red or white LED light sources. A useradjusted brightness feature coupled with a brightness capability exceeding that of the prior Trachlight may allow usage in a broader range of neck girths and clinical situations. It awaits further study.

Acknowledgements The authors thank Ms. Leah M. Wood, MSc for her assistance with the cadaver laboratory testing.

Competing interests All three authors are the co-founders of Scotia MD Engineering Inc which holds the intellectual property rights for the device used in this study (patent pending).

Funding sources The design and development of the prototype device was supported by a National Research Council Canada, Industrial Research Assistance Program (IRAP) grant. The cadaveric laboratory costs were supported by an internal research fund within our Department of Anesthesia, Pain Management and Perioperative Medicine, Dalhousie University.

\section{References}

1. Hung OR, Pytka S, Morris I, Murphy M, Stewart RD. Lightwand intubation: II. Clinical trial of a new lightwand for tracheal intubation in patients with difficult airways. Can. J Anesth 1995; 42: 826-30.

2. Milne AD, Cave AL, Brousseau PA, Hung OR. The effect of sterilisation on the light intensity of the reusable Trachlight ${ }^{\mathrm{TM}}$. Anaesthesia 2012; 67: 40-2.

3. Sawanobori Y, Ueki M, Kagawa T, Maekawa N. A simple modification of the Trachlight for pediatric use. J Anesth 2008; 22: 338-9.

4. Malan CA, Scholz A, Wilkes AR, Hampson MA, Hall JE. Minimum and optimum light requirements for laryngoscopy in paediatric anaesthesia: a manikin study. Anaesthesia 2008; 63: 65-70. 